\title{
The algorithm for MODIS wavelength on-orbit calibration using the SRCA
}

\author{
By Harry Montgomery ${ }^{1}$ Nianzeng Che ${ }^{2}$, Kirsten Parker $^{3}$, and Jeff Bowser ${ }^{4}$ \\ MAs? \\ Plon

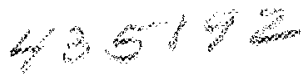

\begin{abstract}
The Spectro-Radiometric Calibration Assembly (SRCA) provides on-orbit spectral calibration of the MODerate resolution Imaging Spectroradiometer (MODIS) reflected solar bands and this paper describes how it is accomplished. The SRCA has two adjacent exit slits: 1) Main slit and 2) Calibration slit. The output from the main slit is measured by a reference silicon photo-diode (SiPD) and then passes through the MODIS. The output from the calibration slit passes through a piece of didymium transmission glass and then it is measured by a calibration SiPD. The centroids of the sharp spectral peaks of a didymium glass are utilized as wavelength standards. After normalization using the reference SiPD signal to eliminate the effects of the illuminating source spectra, the calibration SiPD establishes the relationship between the peaks of the didymium spectra and the grating angle; this is accomplished through the grating equation. In the grating equation the monochromator parameters, $\beta$ (half angle between the incident and diffractive beams) and $\theta_{\text {off }}$ (offset angle of the grating motor) are determined by matching, in a least square sense, the known centroid wavelengths of the didymium peaks and the calculated centroid grating angles from the calibration SiPD signals for the peaks. A displacement between the calibration SiPD and the reference SiPD complicates the signal processing.

The response of the MODIS bands to the SRCA output is also normalized by the reference SiPD signal to eliminate the effect of the source spectrum. That response differs from what is measured by MODIS using the Spectral Measurements Assembly (SpMA), a laboratory double-monochromator, due to the wider slit width of the SRCA. The SRCA slit function, calculated using measurements by the SpMA and the SRCA at the same MODIS temperature, will be used to recover the MODIS spectral response using the SRCA data measured on-orbit.
\end{abstract}

A series of test results, at different temperatures, indicate that the SRCA is stable and is capable of detecting MODIS wavelength shift on-orbit.

1. Goddard Space Flight Center, Greenbelt, MD 20771, hmontgom@itpmail.gsfc.nasa.gov

2. Swales Aerospace, Beltsville, MD 20705, nche@gscmail.gsfc.nasa.gov

3. Research and Data System Co., Greenbelt, MD 20771,kirsten@ltpmail.gsfc.nasa.gov

4. Raytheon STX, Lanham, MD 20706, jeff.bowser@gsfc.nasa.gov 


\section{Introduction}

The Spectro-Radiometric Calibration Assembly (SRCA) is one of the on-board calibrators in MODerate resolution Imaging Spectrometer (MODIS), which is being designed and manufactured by Raytheon Santa Barbara Remote Sensing. The SRCA is capable of radiometric, spectral, and spatial calibrations.

The reasons for on-orbit spectral calibration are: (1) Spectral band responses could change because of variations of the transmissive properties of the thin-film multi-layer filters with temperature [1]. Band shifts were observed during ground testing of precursor remote sensing instruments. Some on-orbit measurements have been interpreted as showing evident shifts in band response profiles. (2) During radiometric calibration of the solar reflective bands, the Sun is the main calibration source for MODIS. The MODIS instrument will be periodically calibrated by viewing an illuminated solar diffuser plate. Knowing the MODIS band shifts helps redefine the incoming solar radiation of the bands. (3) Band response profiles, recovered from the MODIS signals measured under SRCA illumination, lead to an understanding of the changes in the spectral response of MODIS bands.

During spectral calibration the SRCA measures the center wavelength shifts of the MODIS bands. It calibrates MODIS bands 1-19 and 26 covering a wavelength range from visible (VIS), near Infrared (NIR), to short wavelength IR (SWIR).

The SRCA is configured as a monochromator during spectral calibration. The SRCA provides end-to-end calibration because its beam passes through the complete optical train, but it only fills about $1 / 5$ of the MODIS aperture. In order to ensure that the measurement has sufficient Signal-to-Noise Ratio (SNR), the exit slit of the SRCA monochromator has been widened to cover 5\% to $27 \%$ of MODIS band widths depending upon the band. Hence, the center wavelengths measured by the SRCA are biased by the exit slit width effect and the partial aperture filling effect.

However, the SRCA is expected to be a stable source. It can detect the wavelength change in two consecutive calibrations. During the prelaunch period MODIS is spectrally calibrated against a laboratory instrument -- the Spectral Measurements Assembly (SpMA) in an ambient environment. The SpMA is a double-monochromator, which fills the MODIS aperture and hence provides accurate spectra for the MODIS bands. At the same or near the same time and environment, the SRCA is operated. The differences of the center wavelengths measured by the SRCA and SpMA serve as correction values [2]. The correction values, which are the differences between SpMA and the SRCA measurements, are assumed unchanged throughout MODIS life. These correction values are mainly due to the use of a different SRCA slit width. In the other measurement environments, such as in thermal vacuum testing at different temperatures and on-orbit, the SRCA has been and will be run repeatedly. SRCA measured center wavelengths compared with the previous measurements indicate band center wavelength shifts. 
Figure 1 is the optical layout of the SRCA, which consists of three sub-assemblies: a light source, a monochromator, and a folded collimator.

The light source is a Spherical Integration Source (SIS) with six embedded lamps (four $10 \mathrm{~W}$ and two $1 \mathrm{~W}$ ). In the spectral mode only two lamp configurations, first three $10 \mathrm{~W}$ and then one $10 \mathrm{~W}$, are applied to minimize the period of the spectral calibration and to prolong lamp life. The radiant output is monitored by a temperature-controlled silicon photodiode (SiPD) embedded in the SIS (not shown in Figure 1) providing a feedback signal to keep the lamp output constant.

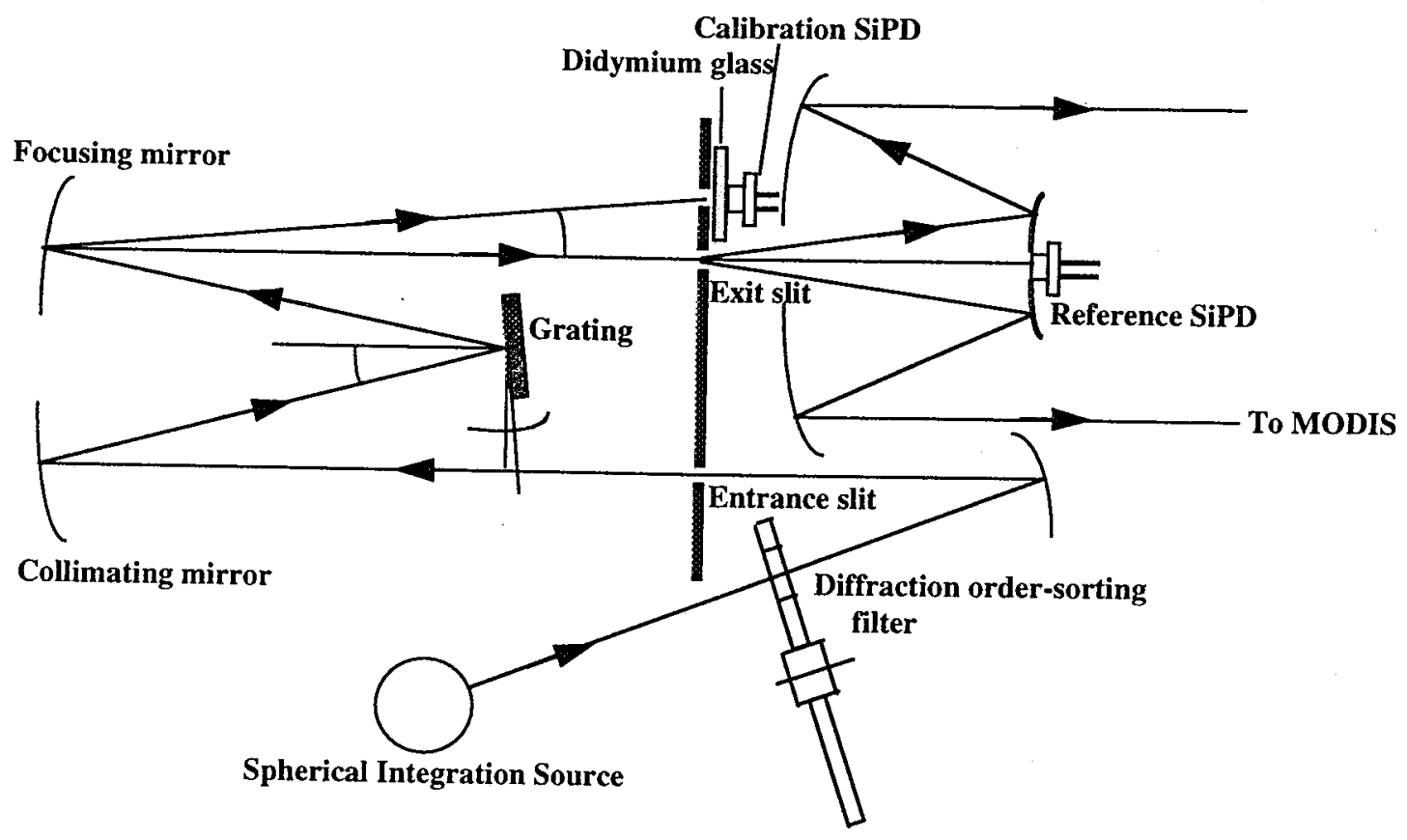

Figure I SRCA layout

To isolate the monochromatic beam from the mix of different diffraction orders, ordersorting filters are inserted into the optical path. A monochromatic beam of different wavelengths is output when the grating rotates with the required order-sorting filters inserted synchronously.

There is a secondary exit slit, which is parallel to and is located six millimeters from the main exit slit, with a didymium glass filter and the calibration SiPD behind it. The spectral beam, from the secondary slit, passes through a didymium glass and is detected by the calibration SiPD while the spectral beam from the main exit slit is sampled by the reference SiPD, with the same characteristics as the calibration SiPD, located at the center of the secondary mirror of the Cassegrain system. The reference and the calibration SiPDs are manufactured in the same lot and selected in pairs. The two sets of signals form the database for wavelength self-calibration. The calibration SiPD signal shape, after normalization by the reference SiPD signal to remove the light source spectral shape, results in a profile which matches the didymium transmission curve. However, the location difference of the two SiPDs complicates the signal processing. 
During the spectral mode heat from the lamps and motors warm the SRCA and the SRCA housing temperature varies with time. In order to compensate for the SiPD spectral sensitivity variation with temperature, the temperatures near the two SiPDs are recorded with the SiPD data.

The MODIS bands spectrally calibrated by the SRCA are listed in Table 1.

Table 1. The center wavelengths and band widths of MODIS bands

\begin{tabular}{|c|c|c|c|c|c|}
\hline Band & Center wavelength $(\mu \mathrm{m})$ & Band width $(\mu \mathrm{m})$ & Band & Center wavelength $(\mu \mathrm{m})$ & Band width $(\mu \mathrm{m})$ \\
\hline 1 & 0.646 & 0.050 & 11 & 0.530 & 0.014 \\
\hline 2 & 0.856 & 0.045 & 12 & 0.547 & 0.012 \\
\hline 3 & 0.466 & 0.021 & 13 & 0.666 & 0.012 \\
\hline 4 & 0.554 & 0.021 & 14 & 0.677 & 0.014 \\
\hline 5 & 1.242 & 0.031 & 15 & 0.747 & 0.013 \\
\hline 6 & 1.629 & 0.036 & 16 & 0.866 & 0.018 \\
\hline 7 & 2.114 & 0.067 & 17 & 0.904 & 0.040 \\
\hline 8 & 0.412 & 0.017 & 18 & 0.936 & 0.016 \\
\hline 9 & 0.442 & 0.011 & 19 & 0.936 & 0.055 \\
\hline 10 & 0.487 & 0.012 & 26 & 1.382 & 0.048 \\
\hline
\end{tabular}

\section{The spectral transmittance of didymium glass and peak location}

Monochromators need to be regularly re-calibrated for wavelength due to possible changes of instrument parameters. A line-spectrum light source is normally utilized as the wavelength calibrator. When the wavelength standard needs to be carried into space, a sharp-peaked absorption glass is a convenient choice. The didymium glass has several absorption and transmission peaks in the desired wavelength range. Although there is no guarantee that the peak location is stable, it is likely to be much more stable than the multi-layer thin-film interference filters used in the MODIS focal plane arrays.

Figure 2 illustrates the spectral transmittance of the didymium glass [3]. The transmission profile has several sharp peaks and valleys. Selection of peaks has the

Transmittance of didymium glass

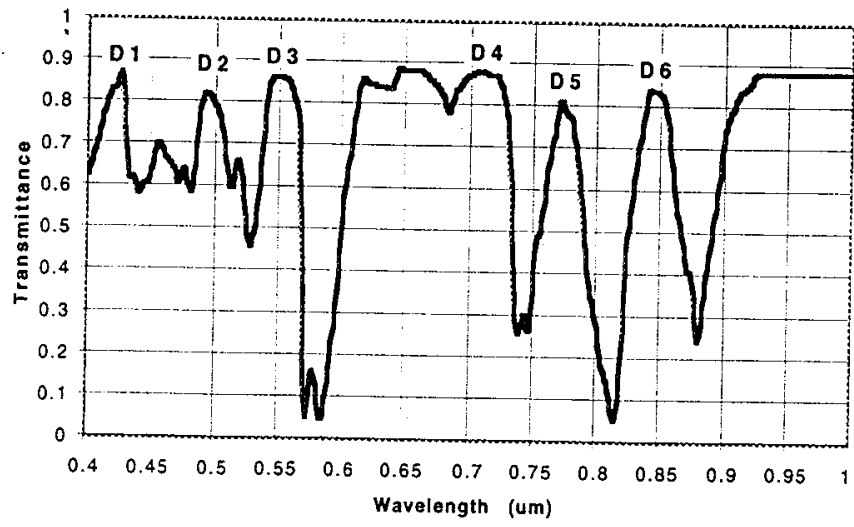

Figure 2. Didymium spectral transmittance advantage of higher SNR in determining the centroid values of the peaks than selection of valleys. The peaks are numbered from D1 to D6 to indicate their locations. The sharper the peaks and the higher the diffraction order applied, the more sensitive the determination of the peak location by the calibration SiPD signal. Two peaks are utilized in this application: one at $0.496 \mu \mathrm{m}$ (D2) and the other at $0.551 \mu \mathrm{m}$ (D3). The selection of the two peaks considers the use of higher diffraction order and the peak locations over the range 
of MODIS bands to be measured. The use of the third diffraction order for $0.496 \mu \mathrm{m}$ $\left(\mathrm{D} 32^{1}\right)$ and $0.551 \mu \mathrm{m}$ (D33) peaks and the second diffraction order for $0.551 \mu \mathrm{m}$ (D23) locates the three peaks in the grating angle range of 6.84 to 12.06 degrees. The entire grating angle range for the calibration spans from 6.35 to 15.33 degrees.

\section{Spectral calibration signal and its normalization}

The basic grating equation is

$$
m \lambda=A\left(\sin \theta_{i}-\sin \theta_{d}\right) \cos \phi
$$

where $A$ is the grating spacing; $m$ is grating order; $\lambda$ is the wavelength of the output beam; $\theta_{\mathrm{i}}=\beta-\theta_{\mathrm{g}}$, is the incident angle about the grating normal in the diffraction plane; and $\theta_{d}=\beta+\theta_{g}$, is the diffraction angle about the grating normal in the diffraction plane. $\theta_{g}$ is the angle between grating normal and the bisector between incident and diffraction rays. $\phi$ is the out of diffraction plane angle.

When the grating rotates an angle of $\theta_{M}$ with an initial offset angle of $\theta_{\text {off }}$, and assuming the beams to be in the diffraction plane for simplicity, the wavelength of the output beam from the main exit slit can be written as

$$
\lambda=\frac{2 A}{m} \sin \left(\theta_{M}+\theta_{o f f}\right) \cdot \cos \beta
$$

if we select negative diffraction orders.

The spectral calibration consists of determining two monochromator parameters: the half angle between the incident beam and the diffraction beam, $\beta$, and the grating motor offset angle, $\theta_{\text {off }}$. The two parameters may change under different operational environments and conditions. As soon as the two parameters are determined using the didymium wavelength standard, the relationship between grating motor angle and wavelength is established and then the monochromator is calibrated.

When the monochromatic beam exits through the didymium slit, an angular distance of $\Delta$ from the main slit, Eq.(2) takes a different form:

$$
\lambda=\frac{2 A}{m} \sin \left(\theta^{\prime}{ }_{M}+\theta_{\text {off }}+\frac{\Delta}{2}\right) \cdot \cos \left(\beta+\frac{\Delta}{2}\right)
$$

In normalization, the signals of the calibration SiPD with the wavelength value of $\lambda$ in Eq. (3) should be divided by the signals of the reference SiPD with the same wavelength value of $\lambda$ in Eq. (2). In other words, the signal from the calibration SiPD at grating angle of $\theta_{M}^{\prime}$ must be divided by that from the reference SiPD at grating angle of $\theta_{M}$. The $\theta^{\prime}{ }_{M}$ corresponding to each $\theta_{M}$ is calculated by setting Eq.(2) equal to Eq.(3). However, since the exact values of $\beta$ and $\theta_{\text {off }}$ are unknown before the calibration, we have to use the design values for the two parameters to relate $\theta_{M}^{\prime}$ to $\theta_{M}$. Since the change of $\beta$ and $\theta_{\text {off }}$ is small, the use of design values of $\beta$ and $\theta_{\text {off }}$ introduce an uncertainty less than one step.

\footnotetext{
${ }^{1}$ D32 means a diffraction order of three and peak number two.
} 


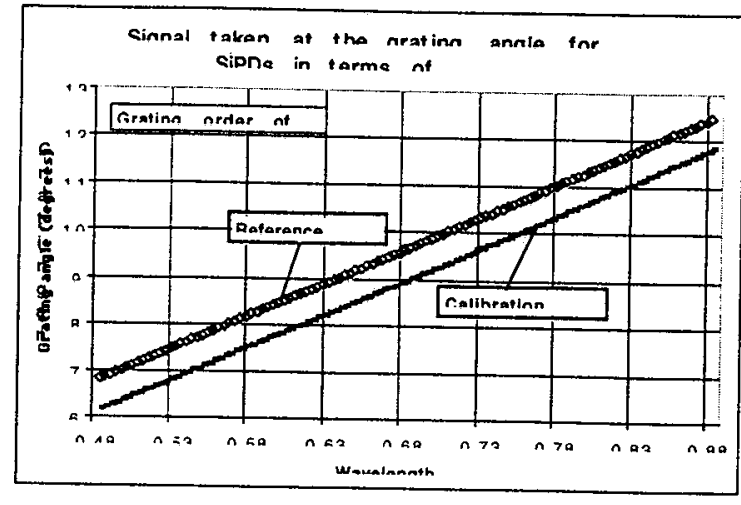

Figure 4. Grating angles $\theta_{M}^{\prime}$ and $\theta_{M}$ in terms of wavelength

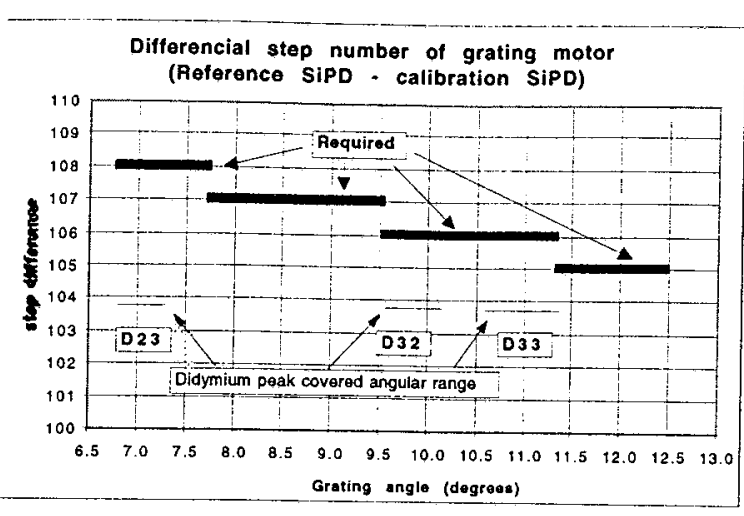

Figure 5. The difference between $\theta_{M}^{\prime}$ and $\theta_{M}$

Figure 4 shows the $\theta^{\prime}$ and $\theta_{M}$ in terms of wavelength according to Eqs. (2) and (3). The two curves are fairly linear and parallel to each other. The difference of $\theta_{M}-\theta_{M}^{\prime}$ in terms of grating motor step is shown in Figure 5. In this application, each step is equivalent to 0.00588 degrees on average. For spectral calibration we are interested only in the grating angle ranges that the didymium peaks cover. The differences $\left(\theta_{M}-\theta_{M}^{\prime}\right)$ for the peaks D23 and D32 are constant (108 and 106 steps, respectively) while the peak D33 has a difference of mostly 106 and partly 105 steps. Considering that one step difference for partial didymium peak sampling does not introduce detectable error, the difference for peak D33 utilizes the value of 106.

Table 2. Grating motor step number for the three didymium peaks [4]

\begin{tabular}{|c|c|c|c|c|c|c|}
\hline \multicolumn{3}{|c|}{$\begin{array}{c}\text { Didymium peaks } \\
\text { Number }\end{array}$} & $\begin{array}{c}\text { Wavelength } \\
(\mu \mathrm{m})\end{array}$ & $\begin{array}{c}\text { Diffraction } \\
\text { order }\end{array}$ & $\begin{array}{c}\text { Motor step for the } \\
\text { didymium slit }\end{array}$ & \multicolumn{2}{|c|}{$\begin{array}{c}\text { Motor step for the } \\
\text { main slit }\end{array}$} \\
\hline D23 & 0.551 & 2 & 31764 & 31857 & 31872 & 31965 \\
\hline D32 & 0.496 & 3 & 32322 & 32334 & 32328 & 32440 \\
\hline D33 & 0.551 & 3 & 32408 & 32544 & 32514 & 32650 \\
\hline
\end{tabular}

Table 2 gives the actual motor step number and the range for the three didymium peaks. The grating motor angle is nominally zero at the grating motor step number of 30600 . Their differences, dstep\#, are 108, 106, and 106 as mentioned. The normalized calibration SiPD signal, $\mathrm{CDS}_{\mathrm{Norm}}$, over the didymium peak region is

$$
C D S_{\text {Norm, }, p k}(\text { step\#, } m)=\frac{D N_{\text {calibration SiPD, L, pk }}(\text { step\#, } m)-D N_{\text {calibration SiPD, dark }}}{D N_{\text {reference SiPD, L, pk }}(\text { step\# }+ \text { dstep\#, } m)-D N_{\text {reference SiPD,dark }}}
$$

where the subscript $\mathrm{L}$ is lamp configuration, 'pk' stands for the peak region, and $\mathrm{m}$ is the diffraction order. Dark readings are taken before the SRCA is turned on and after the SRCA lamps are turned off. Test data have shown that the dark reading is stable throughout the calibration period, an average value is assigned. 
Figure 6 shows the signals of the calibration SiPD and the reference SiPD, both with dark voltage subtracted, versus grating motor step number. The locations of the three peaks are marked by arrows. The other reference SiPD data are applied for normalization of MODIS band/channel signal while the calibration SiPD data are useful only in the vicinity of the didymium peaks. Figure 7 shows the normalized calibration SiPD signal in terms of grating motor angle only in the ranges listed in Table 2.

Before the reference SiPD signal is used to normalize the calibration SiPD signal, we have to handle the noise of the reference SiPD signal. The noisy reference SiPD signal is attributed to the high gain setting of the SiPD circuit. If the noise is not eliminated all calibration signals and MODIS band response profiles will be noisy after these signals are divided by the reference SiPD signals. A quadratic equation fits the measured reference SiPD to smooth out the noise without degrading the centroid value of the reference SiPD signal. Figure 8 shows the measured reference SiPD signal and the noisesmoothed reference SiPD profiles for $30 \mathrm{~W}$ at Nominal temperature plateau (269K). Spurious (obviously noisy) signals have been excluded from the calibration.

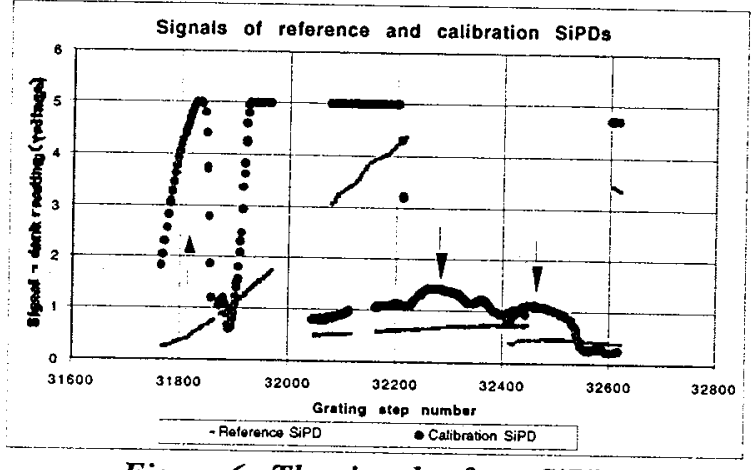

Figure 6. The signals of two SiPDs

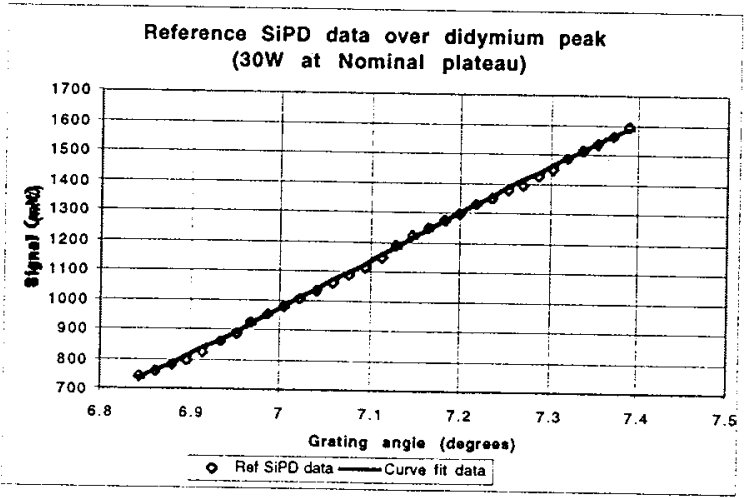

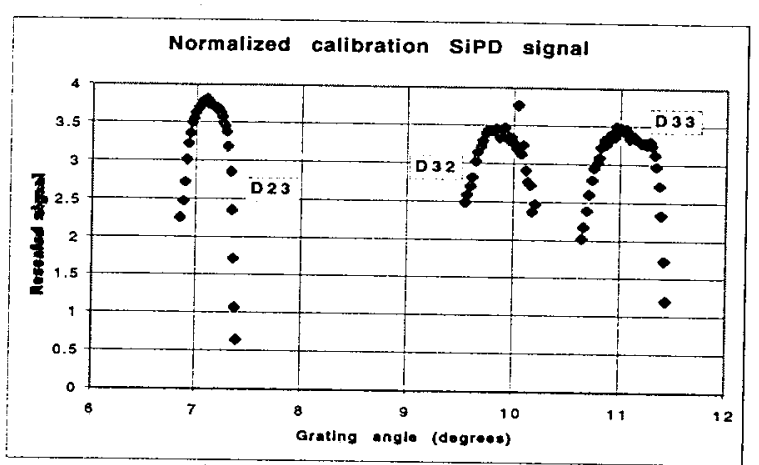

Figure 7. Normalized calibration SiPD signals

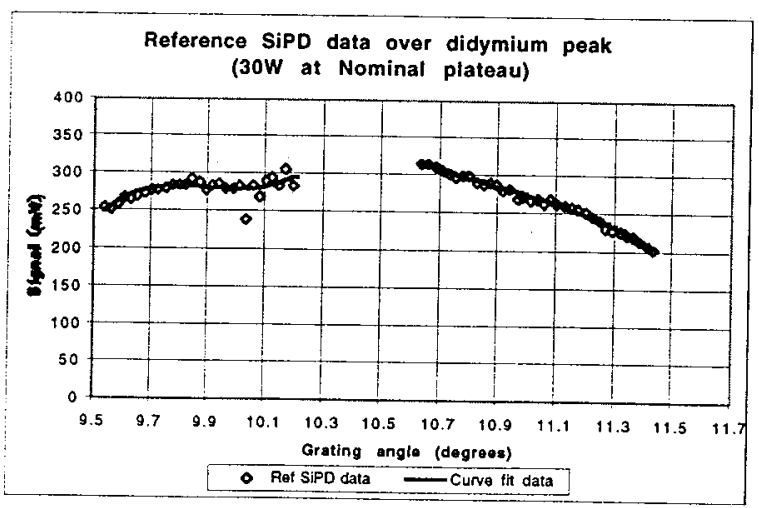

Figure 8. Polynomial approximation to smooth out noise of the reference SiPD signal

\section{Spectral calibration signal to cover all MODIS reflected solar bands}

To obtain adequate SNR levels for the 20 MODIS bands to be calibrated, two lamp configurations are applied: $1-10 \mathrm{~W}$ and 3-10W. The motor rotates the grating 
unidirectionally to avoid uncertainty due to motor backlash or motor positioning. For the $30 \mathrm{~W}$ lamp configuration, the grating rotation covers MODIS bands $1-4,8-11$, and 17 plus the three didymium peaks. Then the grating rotates to its home position. For $1-10 \mathrm{~W}$ lamp configuration, one unidirectional rotation of the grating covers wavelengths for MODIS bands $5-7,12-16,18,19$, and 26 plus the three didymium peaks. The two monochromator parameters, $\beta$ and $\theta_{\text {off }}$, are determined for each lamp configuration to account for the grating motor starting at a different offset angle when it rotates to its home position. The offset angle, $\theta_{\text {off }}$, is critical to the calibration accuracy.

The order-sorting filter mounted on the filter wheel is positioned according to the diffraction order required and grating angle (Table 3).

Table 3. The relationship of diffraction order with filter\# and grating angle [2]

\begin{tabular}{|c|c|c|}
\hline Filter\# & Grating angle (degree) & Diffraction order \\
\hline open hole & $7.8-15.0$ & -1 \\
\hline$\# 1$ & $7.9-15.0$ & -3 \\
\hline$\# 2$ & $<10.4$ & -2 \\
\hline$\# 2$ & $>10.4$ & -3 \\
\hline$\# 3$ & $<8.6$ & -1 \\
\hline$\# 3$ & $>8.6$ & -2 \\
\hline
\end{tabular}

Note: The band pass is $0.40-0.52 \mu \mathrm{m}$ for Filter $\# 1,0.52-0.70 \mu \mathrm{m}$ for Filter $\# 2$, and $0.73-0.99 \mu \mathrm{m}$ for Filter\#3.

The recovery of 20 MODIS band responses and six didymium peaks in two unidirectional grating rotations requires that the grating motor start step\#, ending step\#, step size, and number of samples per collection be carefully designed. Even in this circumstance some bands may have to share the same grating position but need different diffraction orders. Hence, the order-sorting filters have to flip back and forth at the same grating step.

The spectral calibration has to be executed when the earth and atmosphere are in dark to eliminate external-illumination effects on the high-gain reference SiPD signal. Under this constraint, the entire calibration has been divided into four periods: two periods per lamp configuration. Each period will be completed within the duration of the dark portion of the orbit ( 35.4 minutes). The operational procedure is executed according to a series of programmed instructions [4].

\section{Determination of the two monochromator parameters, $\beta$ and $\theta_{\text {off }}$}

The angular displacement of $\Delta$ in Eq.(3) is calculated from

$$
\Delta=\tan ^{-1}\left(\frac{d S}{f_{\text {mono }}}\right)
$$

where dS is the distance between the main exit slit and the didymium slit $(6 \mathrm{~mm})$, and $\mathrm{f}_{\text {mono }}(260.6 \mathrm{~mm})$ is the focal length of the monochromator. It seems in Eq. (3) that the angular displacement could be another parameter so that three monochromator parameters are uniquely determined by the three peak positions. However, it does not 
behave that way. Notice that the parameters $\beta$ and $\theta_{\text {off }}$ both have $\Delta / 2$ added to them. An increment of $\Delta / 2$ merely leads to corresponding reductions in $\beta$ and $\theta_{\text {off }}$ by the same amount. It means $\Delta / 2$ can not be determined as an independent parameter. We use the design value of $\Delta / 2$. Variation in the monochromator focal length will introduce uncertainty to the calibration.

The monochromator parameters, $\beta$ and $\theta_{\text {off }}$, are derived by best matching the didymium peaks in terms of wavelength and the normalized calibration SiPD signal in terms of grating motor angle. The match is not the detailed profile but the centroid values of these peaks. Actually peak D3 (Figure 2) at $0.551 \mu \mathrm{m}$ is used with both diffraction orders 2 and 3 ; these two combinations occur at two distinct grating motor angles. Hence, the two didymium peaks lead to three unique grating angles and also three distinct centroid wavelengths because of the possible difference in the number of samples which are used to compute the centroids for the same peak. That means we relate the three centroid wavelength values of the two didymium peaks to the centroid values of the normalized calibration SiPD signal for the three peaks through the grating equation.

After conversion of grating motor step number to angle using the database, the normalized calibration signal becomes $\operatorname{CDS}_{\text {norm, pk }}\left(\theta_{M}^{\prime}, m\right)$. The centroid value of the normalized SiPD signal for each peak, $\Omega_{m, p k, L}^{c D s}$ (degrees), is calculated by

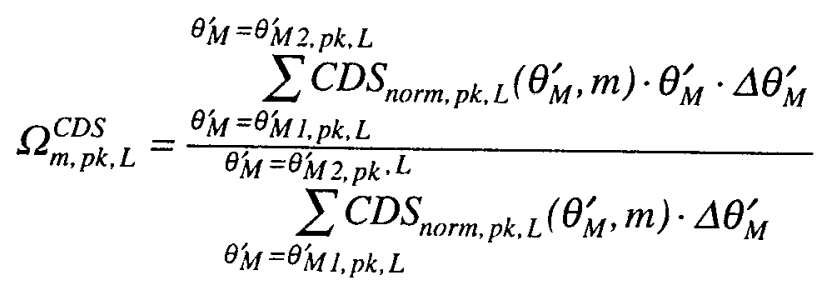

The data points are limited by a threshold value, which equals to $0.7 \mathrm{CDS}_{\text {norm,pk,L,max }}$. Setting the threshold maintains the consistency of the calibration results. In Eq.(6), the $\theta^{\prime}{ }_{M 1}$ and $\theta_{M 2}^{\prime}$ are both wings of the angle values with the signal intensity greater than the threshold. The number of data points depends on data availability.

The centroid wavelength of the didymium transmission peak is calculated over the wavelength region of $\lambda_{1, \mathrm{pk}, \mathrm{L}}$ and $\lambda_{2, \mathrm{pk}, \mathrm{L}}$, which correspond to $\theta_{\mathrm{M1}, \mathrm{pk}, \mathrm{L}}^{\prime}$ and $\theta_{\mathrm{M} 2 \mathrm{pk}, \mathrm{L}}^{\prime}$ and are calculated by Eq.(3) with design values of $\beta$ and $\theta_{\text {off. }}$ Sampling for the centroid calculation over the angle range of Eq.(6) is not necessarily equally spaced. If some bands have the same diffraction orders and are sampled in the peak region with different step size, more data points may be available and used in Eq.(6). If we ignore the situation and calculate the centroid wavelength of the didymium peak with a constant step size, a bias could be introduced and the calibration may be unstable due to the inconsistency in data sampling. The centroid wavelength of the didymium peak is 


$$
\lambda_{\text {didymium }, p k, L}=\frac{\sum_{\lambda=\lambda_{I, p k, L}}^{\lambda=\lambda_{2, p} k, L} \tau_{\text {didymium }}(\lambda) \cdot \lambda \cdot \Delta \lambda}{\sum_{\lambda=\lambda_{2, p k, L}} \tau_{\text {didymium }}(\lambda) \cdot \Delta \lambda}
$$

where $\tau_{\text {didymium }}(\lambda)$ is the spectral transmittance of the didymium glass. Because different lamp configurations have different data stepping strategies, the centroid wavelength of $\lambda_{\text {didymium,pk.L }}$ must be calculated for each individual lamp configuration.

A least-square fit technique [5] to adjust $\beta_{\mathrm{L}}$ and $\theta_{\text {off, } \mathrm{L}}$ until the $\lambda_{p k, L}^{C D S}$ best fits $\lambda_{\text {didymium,pk,L }}$ at a number of peaks, $N_{p e a k}$, is in use. $\lambda_{p k, L}^{C D S}$ is computed by substituting $\Omega_{m, p k, L}^{C D S}$ into Eq.(3) for $\theta_{M}^{\prime}$

Starting with the grating equation (3),

$$
m \lambda_{\text {didymium, } p k, L}=m \lambda_{p k, L}^{C D S}=a_{1 L} \cdot \sin \Omega_{m, p k, L}^{C D S}+a_{2 L} \cdot \cos \Omega_{m, p k, L}^{C D S}
$$

where $a_{1 L}$ and $a_{2 L}$ are the function of $\beta_{L}$ and $\theta_{o f f, L}$ :

$$
\begin{gathered}
a_{1 L}=2 A \cdot \cos \left(\theta_{o f f, L}+\Delta / 2\right) \cdot \cos \left(\beta_{L}+\Delta / 2\right) \\
a_{2 L}=2 A \cdot \sin \left(\theta_{o f f, L}+\Delta / 2\right) \cdot \cos \left(\beta_{L}+\Delta / 2\right)
\end{gathered}
$$

The result of the linear fit using the three sets of $\Omega_{m, p k, L}^{C D S}$ and $m \lambda_{\text {didymiu,pk,L }}$ in Eq.(8) gives the best predicted values of $a_{1 L}$ and $a_{2 L}$. From the Eqs.(9) and (10), the two monochromator parameters can be calculated.

$$
\begin{aligned}
& \theta_{o f f, L}=\tan ^{-1}\left(\frac{a_{2 L}}{a_{l L}}\right)-\frac{\Delta}{2} \\
& \beta_{L}=\cos ^{-1}\left(\frac{a_{l L}}{2 A \cos \left(\theta_{o f f . L}+\frac{\Delta}{2}\right)}\right)-\frac{\Delta}{2}
\end{aligned}
$$

Figure 9 shows the match of normalized calibration signals with didymium peaks at three grating motor regions. The two parameters calculated at different temperature plateaus are listed in Table 4 . The results are highly consistent. 


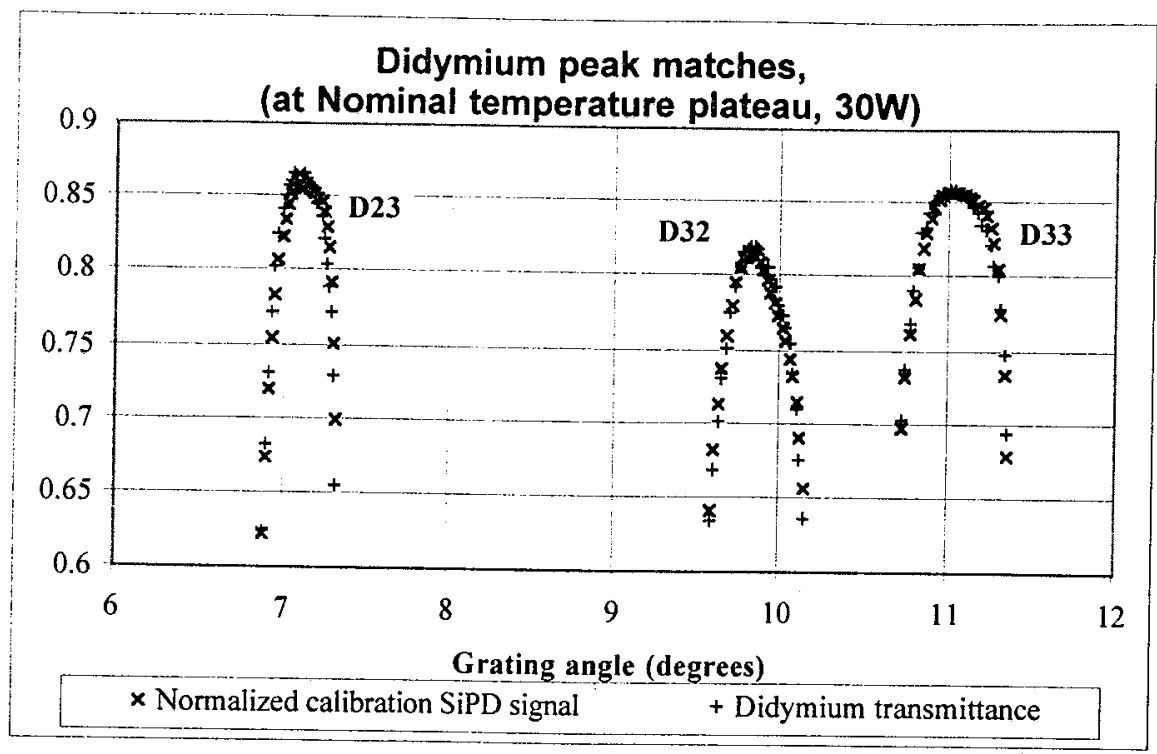

Figure 9. Match the centroid values of didymium peaks with that of normalized calibration

Table 4. Monochromator parameters measured in eight calibrations

\begin{tabular}{|c|c|c|}
\hline & $\beta$ (half angle) & $\theta_{\text {orr }}$ (grating motor offset angle) \\
\hline Nominal value & $15^{\circ}$ & $0^{\circ}$ \\
\hline $30 \mathrm{~W}$, Nominal plateau (269K) & $15.053^{\circ}$ & $0.006^{\circ}$ \\
\hline $10 \mathrm{~W}$, Nominal plateau (269K) & $15.108^{\circ}$ & $0.003^{\circ}$ \\
\hline $10 \mathrm{~W}$, Hot plateau (279K) & $15.059^{\circ}$ & $0.002^{\circ}$ \\
\hline $10 \mathrm{~W}$, Cold plateau (253K) & $15.076^{\circ}$ & $0.006^{\circ}$ \\
\hline $30 \mathrm{~W}$, ambient (1) & $15.030^{\circ}$ & $0.000^{\circ}$ \\
\hline $10 \mathrm{~W}$, ambient (1) & $15.002^{\circ}$ & $-0.001^{\circ}$ \\
\hline $30 \mathrm{~W}$, ambient (2) & $15.035^{\circ}$ & $0.000^{\circ}$ \\
\hline $10 \mathrm{~W}$, ambient (2) & $15.045^{\circ}$ & $0.001^{\circ}$ \\
\hline
\end{tabular}

\section{Handling dark readings for subsample data}

Three detector sizes are used in the MODIS focal plane assemblies (FPAs): $0.25 \mathrm{~km}$ IFOV (Instantaneous Field of View) for bands $1-2 ; 0.5 \mathrm{~km}$ IFOV for bands $3-7$, and $1 \mathrm{~km}$ IFOV for band numbers greater than 7. Accordingly, the sample rate varies for different detector sizes. The SRCA has ten frames (one frame equals to $1 \mathrm{~km}$ IFOV) of data. Hence, there are four subsamples for each channel of bands 1 - 2 (for a total of 40 samples per scan per channel), two subsamples for bands 3 - 7 (for a total of 20 samples per scan per channel), and one sample for band number greater than 7 (10 samples per scan per channel).

The dn, digital counts after space counts is subtracted, may vary between subsamples because of the possible differences in subsamples such as integration time. Figure 10 illustrates an example of the dn variation between subsamples. Since only one fully illuminated sample is available, this sample is picked as the signal. Notice that the dark ${ }^{2}$ readings vary from subsample to subsample. Sample\#10 falls into the subsample\#2

\footnotetext{
${ }^{2}$ The dark values in Figure 10 are those for which no light through the slit falls on the detector.
} 
(even sample) category, for which the dark dn is smaller than that for subsample\#1 (odd sample).

The dn signal must correspond to the sample where the detector is fully illuminated by the slit. It appears at sample\#10 in Figure 10. When the signal dn (sample\#10) is picked up for each individual step, the dark dn is calculated by averaging over several nonilluminated samples with the same subsample number as the signal $\mathrm{dn}$. We define it as $\mathrm{dn}^{\prime}, \mathrm{dn}-\mathrm{dn}_{\mathrm{dark}}$. Figure 11 shows $\mathrm{dn}^{\prime}$ versus grating motor step number for that band. ${ }^{3}$

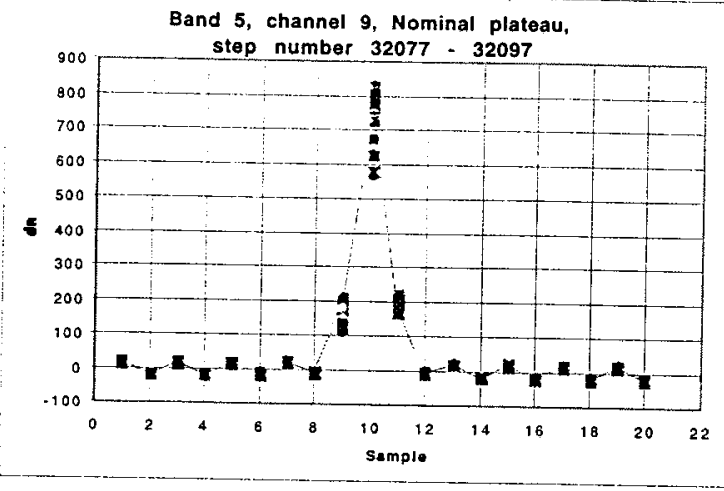

Figure 10. Data sample in scans

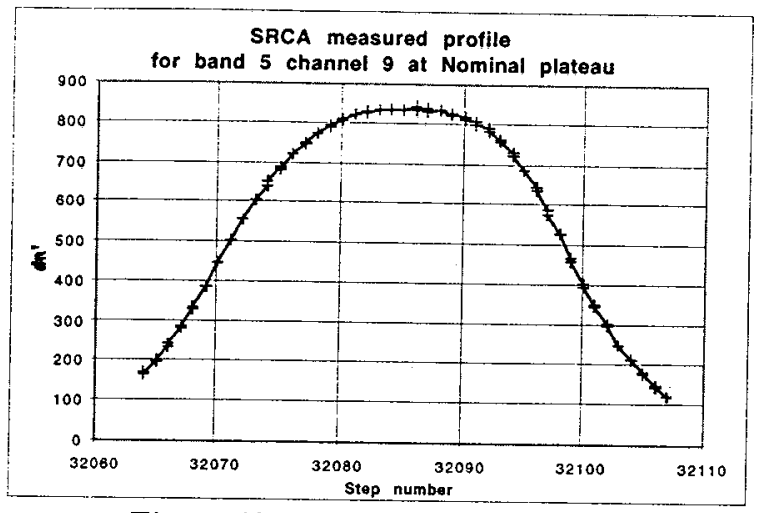

Figure 13. SRCA measured band response using $\mathrm{dn}^{\prime}$

\section{The center wavelengths of the MODIS bands}

The MODIS bands respond to the SRCA monochromatic beam, which is spectrally shaped by the light source, grating efficiency, and the SRCA system transmittance. This shape must be eliminated from the measurement. The reference SiPD signal provides this capability. Notice that the reference SiPD signal contains not only the spectral shape of the lamp source but also the spectral response, $R_{\text {SDS }}(\lambda)$. Hence, the normalized MODIS band response, $\mathrm{R}_{\text {band,channel.Nom }}(\lambda)$, must be divided by the reference $\operatorname{SiPD}$ signal, $\operatorname{SDS}(\lambda)$, and multiplied by the SiPD spectral response.

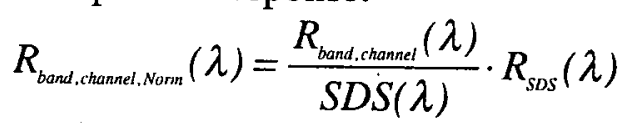

Next the subscript, channel, is defined as follows: There is a communication channel or data channel corresponding to each detector in each MODIS spectral band; bands 1 and 2 each have 40 channels; bands 3-7 each have 20 channels; and bands 8-19 and 26 each have 10 channels. Limited by the SiPD response cut-off wavelength at about $1.1 \mu \mathrm{m}$, the SWIR bands $5(1.242 \mu \mathrm{m}), 6(1.629 \mu \mathrm{m}), 7(2.115 \mu \mathrm{m})$, and $26(1.382 \mu \mathrm{m})$ can not be normalized by the reference SiPD signal. Fortunately, this does not cause evident uncertainty in the calibration for these bands because: (1) the SRCA detects the center wavelength shift. The detection is a comparison of calibration results from prelaunch and on-orbit, hence, the normalization effect will be significantly reduced. (2) The wavelength of the SWIR is located where the lamp output profile changes slowly with

\footnotetext{
${ }^{3}$ It is anticipate that the on-going improvement will significantly reduce the differences. However, this algorithm will still be used.
} 
lamp temperature. According to data analysis, a $10 \mathrm{~K}$ change in the lamp temperature will cause the SRCA source profile to vary by only $0.8 \%$ and the center wavelength will change by only $0.03 \mathrm{~nm}$.

The MODIS center wavelength is calculated by

$$
\lambda_{c}(\text { band }, \text { channel })=\frac{\sum_{\lambda=\lambda_{1}}^{\lambda=\lambda_{2}} \frac{d n^{\prime}(\text { band, channel }, \lambda)}{R_{\text {band, channel, Norm }}(\lambda)} \cdot \lambda \cdot \Delta \lambda}{\sum_{\lambda=\lambda_{1}}^{\lambda=\lambda_{1}} \frac{d n^{\prime}(\text { band, channel, } \lambda)}{R_{\text {band, channel, Norm }}(\lambda)} \cdot \Delta \lambda}
$$

where the SRCA calibration results relate the wavelength, $\lambda$, to the grating motor step number.

The SRCA measured MODIS band/channel response profiles are modified by the wide slit width so that the spectral responses measured by the SRCA differ from those measured by the SpMA. The SRCA slit width is equivalent to $5 \%-27 \%$ of MODIS bandwidths depending on the band width and the diffraction order. Figure 12 gives an example of band response measured by the two instruments. The SRCA measured center wavelength is biased, but, the SRCA measured center wavelength shift between any two measurements determines the MODIS band shift because the SRCA slit size is fixed and limited variation in system characteristics do not change the center wavelength value. The differences between the center wavelengths measured by the SpMA and the SRCA in the same environment are the SRCA wavelength correction values, $d \lambda_{c, b a n d, \text { channel }}$ (Eq.15). The MODIS center wavelengths measured over the MODIS lifetime, $\lambda_{c, \text { band,channel,on-orbit }}$, are calculated by adding the correction values to the current measured center wavelength, $\lambda_{\mathrm{c}, \mathrm{SRCA} \text {, band,channel,on-orbit }}(\mathrm{Eq} .16)$, assuming that the correction values do not change.

$$
\begin{gathered}
d \lambda_{c, \text { band,channel }}=\lambda_{c, S p M A, \text { band,channel }}-\lambda_{c, S R C A, \text { band.channel,prelaunch }} \\
\lambda_{c, \text { band,chunnel,on-orbit }}=\lambda_{c, S R C A, \text { band,channel,on-orbit }}+d \lambda_{c, \text { band,chunnel }}
\end{gathered}
$$

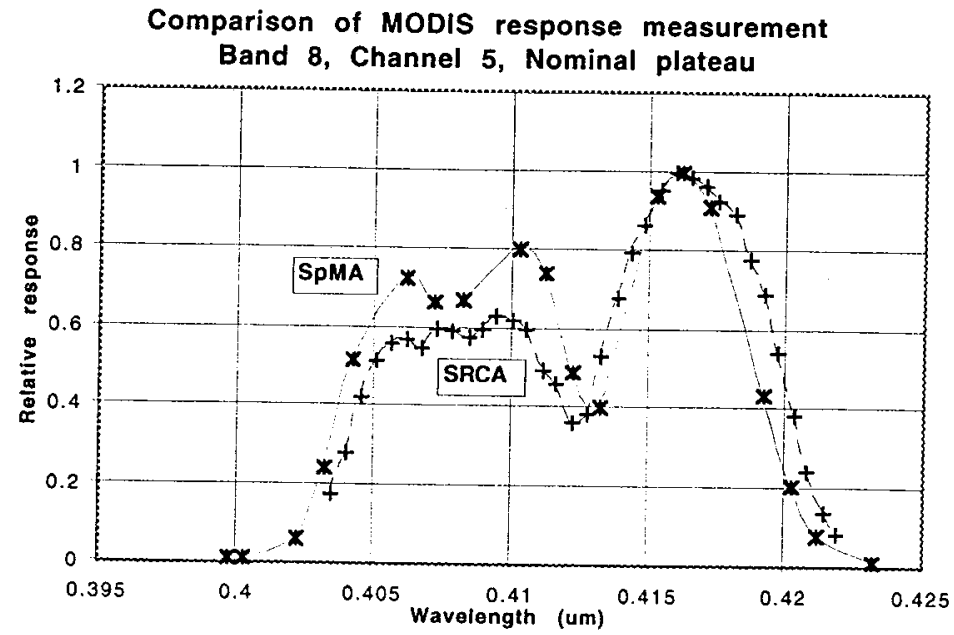

Figure 12. Difference of measured band responses by the SRCA and SpMA 


\section{Recovery of MODIS band/channel relative spectral response profiles from SRCA measurements}

Recovery of MODIS band/channel relative spectral response is possible if the SRCA slit function does not change over time.

The recovery methodology is to calculate the SRCA slit function by dividing the Fourier transform of the MODIS measured response to the SRCA by the Fourier transform of the MODIS measured response to the SpMA at nearly the same time and with the same measurement environment temperature. The light source distribution for the SRCA has been normalized by using the reference SiPD signal (see Eq.(13)); and the SpMA light source distribution has also been normalized by using measurements from a sensor of known response. Therefore, we can assume that the Fourier transform of the slit function of the double-monochromator, SpMA, is unity. The Fourier Transform of the SRCA slit function, $S(\omega)$, is

$$
S(\omega)_{\text {band,channel }}=\frac{F\left(\operatorname{SRCA}(\lambda)_{\text {prelaunch, band, channel }}\right)}{F\left(\operatorname{SpMA}(\lambda)_{\text {prelaunch, band, channel }}\right)}
$$

where the operator, $\mathrm{F}$, is the Fourier Transform.

This slit function (mainly depending on the slit width, grating status, stray-light level, and scattering) is assumed unchanged. The MODIS band/channel relative response is calculated using the SRCA measured profiles on-orbit by

$$
\text { Band response }(\lambda)_{\text {onorbit,band, channel }}=F^{-l}\left(\frac{F\left(\operatorname{SRCA}(\lambda)_{\text {onorbit, band, }, \text { hannel }}\right)}{S(\omega)_{\text {band,channel }}}\right)
$$

Figure 13 illustrates the band response of MODIS band 8 channel 2 measured by the SpMA (left) and by the SRCA at Nominal temperature plateau (right) and recovered using the Eqs. (17) and (18).
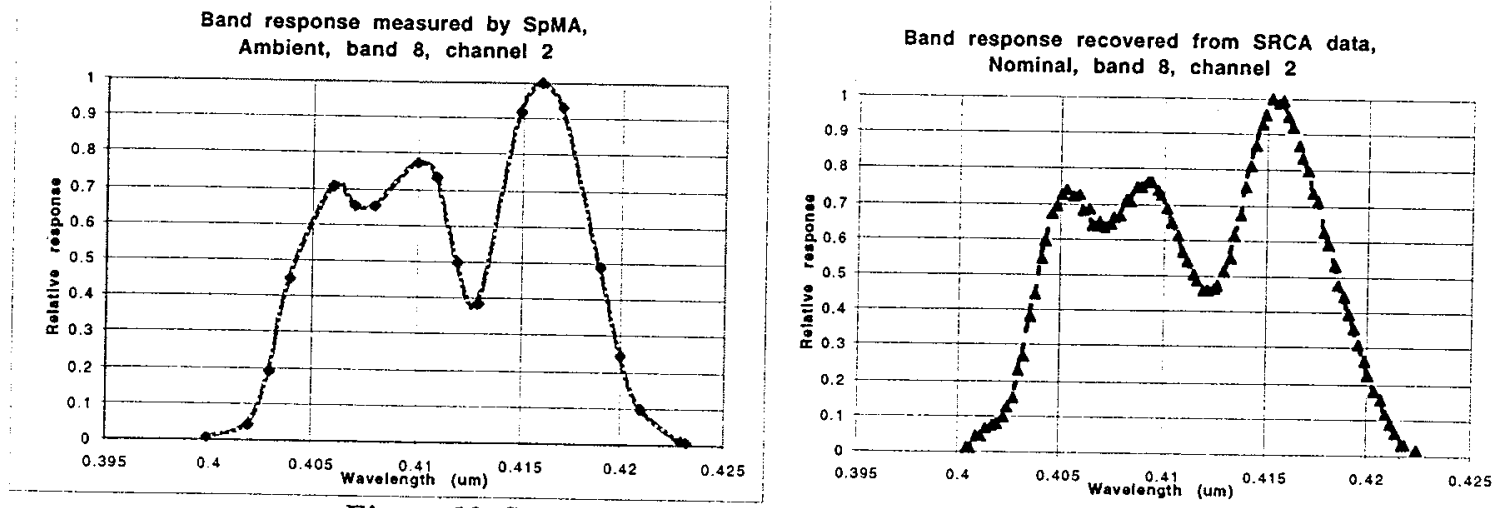

Figure 13. Spectral response recovery for band 8 channel 2

\section{Uncertainty of the spectral calibration}

Based on the measurement data, the main uncertainty sources are summarized as follows:

(1) The uncertainty of grating spacing contains two aspects: the spacing variation with temperature and the spacing repeatability in manufacturing. Low expansion material 
limits the uncertainty to less than $0.2 \mathrm{~nm}$ for temperature change of $\pm 20 \mathrm{~K}$. The spacing repeatability is better than $0.01 \mu \mathrm{m}$, which causes uncertainty of less than $0.1 \mathrm{~nm}$ [6].

(2) The grating motor angle in terms of step number shows a variation of less than a single step $\left(0.00588^{\circ}\right.$ per step on average) [7]. The centroid calculation from more than 29 data points significantly reduces the effect of the angular variation.

(3) The uncertainty of monochromator parameters, $\beta$ and $\theta_{\text {off }}$, depends mainly on the number of data points, the noise of the two SiPDs, and the SiPD response variation with temperature. The number of samples over each didymium peak is between $29-35$. The noise of the SiPD comes from possible saturation and noisy reference SiPD signal due to the high gain setting. The temperature correction value for the SiPD response should be determined based on the manufacturer's temperature correction.

Some MODIS centroid wavelengths can be determined for both lamp configurations $(10 \mathrm{~W}$ and $30 \mathrm{~W})$. Evaluation for this uncertainty source is from the centroid wavelength difference between the two calibrations at the same temperature plateau.

(4) The focal length, $f_{\text {mono }}$, varies with temperature and/or other factors, which leads to a variation of angular displacement, $\Delta$. The uncertainty has high weight to the spectral uncertainty. If the focal length changes less than $1 \%$, the uncertainty contributes the same order of uncertainty as the grating motor offset angle.

(5) Reference SiPD noise is due to the high gain setting. The algorithm discards the bad data points and smoothes the SiPD output profiles.

(6) SiPD spectral response changes with temperature. The temperatures are synchronously measured with SiPD data. Correction for this variation is part of the algorithm.

(7) No normalization for MODIS signals of SWIR bands $(\lambda>1 \mu \mathrm{m})$. As previously mentioned in section 7, this uncertainty is small.

The estimated uncertainty of the spectral calibration (precision) is given in Figure 14. It indicates that the uncertainty is less than the specification required for the precision inflight calibration: $0.5 \mathrm{~nm}$ for the shortest wavelength band $(0.412 \mu \mathrm{m})$ and scaled for wavelength greater up to $1 \mu \mathrm{m}$ [8]. In Figure 14 all channels are shown for each band.

\section{Concluding remarks}

The SRCA is a unique on-board, self-calibrated, spectral source. A didymium glass transmission filter serves as a wavelength standard and the wavelength of the output monochromatic beam is scaled by determining the monochromator parameters in each run of the calibration. Measurements under different temperature environments indicate that high repeatability can be expected. The precision of the wavelength calibration meets the requirement of the MODIS specification. 


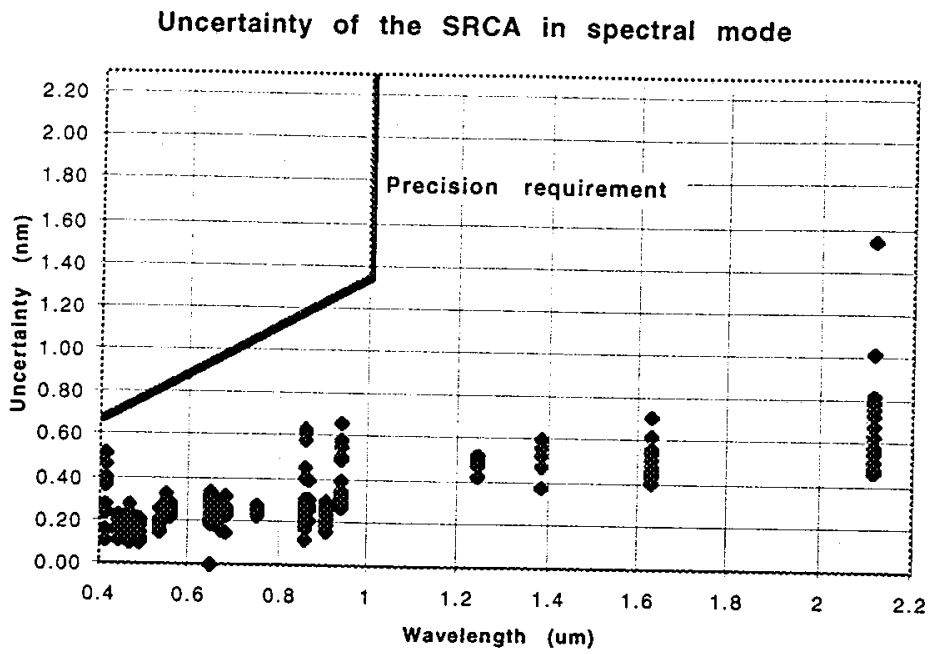

Figure 14. Precision of the SRCA spectral calibration

\section{Acknowledgment}

The authors wish to thank SBRS for the use of related data files. Special thanks are given to J. Young, E. Johnson, T. Paggano, and R. Osgood of SBRS for the beneficial discussion. This work is supported by NASA Contract number NAS5-32650.

\section{$\underline{\text { References }}$}

[1] "The cyro-testing of infrared filters and beamsplitters for the Cosmic Background Explorer's instruments", J. Heaney, K. Stewart, R. Boucarut, P. Alley, and A. Korb, SPIE vol. 619, 1986, p.142-147.

[2]"SRCA calibration algorithms", SBRS Internal Memorandum, PL3095-Q03146, by E. Johnson, Oct. 1993.

[3] Data measured by SBRS, 1997.

[4] "MODIS operations concept document [M0007]", version 2.0, by G. Hatten, C. Salo, K. Parker, E. Knight, and R. Sabatino, Sep.1997.

[5] "SRCA spectral calibration algorithm", SBRS Internal Memorandum, PL3095Q05661, by R. Osgood, March. 1996.

[6] "SRCA spectral measurement accuracy", SBRC Internal Memorandum, PL3095Q02020, by E. Johnson, Jan. 1993.

[7] "SRCA grating motor data", SBRS Internal Memorandum, PL3095-Q05520, by E. Johnson, Nov. 1995.

[8] "Specification for the MODerate-resolution Imaging Spectroradiometer (MODIS)", EOS-AM project, GSFC 422-20-02, Mar. 1993. 